\title{
Active Stateful PCE With Hitless LDPC Code Adaptation [Invited]
}

\author{
F. Cugini, F. Fresi, F. Paolucci, G. Meloni, N. Sambo, A. Giorgetti, \\ T. Foggi, L. Potí, and P. Castoldi
}

\begin{abstract}
The path computation element (PCE) architecture was originally proposed with a stateless condition, i.e., considering only network reserved resources during constraint-based path computations. More recently, a stateful architecture was introduced to additionally maintain the state of computed and established label switch paths (LSPs). Furthermore, the PCE architecture evolved to active functionality, enabling the PCE to directly issue recommendations to the network. In this study, we present and discuss several use cases where the active stateful architecture can provide some benefits. They include impairmentaware path computations in the context of multirate optical networks, recovery solutions, global defragmentation, and dynamic LSP adaptations. The latter use case is then specifically demonstrated in a network testbed including a flexigrid optical network operated with a multicarrier $1 \mathrm{~Tb} / \mathrm{s}$ transmission with coherent detection. Novel advanced digital signal processing (DSP) monitoring functionalities are introduced and experimentally demonstrated. These monitoring functionalities are utilized to trigger a new hitless dynamic adaptation technique operating on the applied low-density parity check (LDPC) transmitted coding. The technique has been successfully demonstrated to increase transmission robustness upon impairment degradation, such that no traffic disruption is experienced. Moreover, to accommodate the LSP coding adaptation, network reconfiguration has been performed, successfully driven by the PCE thanks to the active functionality.
\end{abstract}

Index Terms-Active stateful PCE; Code adaptation; DSP; FEC; Low-density parity check (LDPC); Monitoring; OAM; Path computation element; PCE; PCEP; Stateless.

\section{INTRODUCTION}

$\mathbf{T}$ he path computation element (PCE) architecture has been proposed to enable effective constraint-based path computations [ $\underline{1}-\underline{3}]$. Relevant studies and solutions have been focusing on the evaluation and improvements of PCE architecture and performance [4-11]. In [12], a survey of the PCE architecture is presented. Additional recent studies on PCE include [13-15]. So far, the PCE architecture has been implemented mainly with a stateless architecture. A stateless PCE relies on a traffic engineering

Manuscript received July 7, 2014; accepted October 19, 2014; published December 4, 2014 (Doc. ID 216352).

F. Cugini (e-mail: filippo.cugini@cnit.it), G. Meloni, T. Foggi, and L. Potí are with CNIT, Italy.

F. Paolucci, F. Fresi, N. Sambo, A. Giorgetti, and P. Castoldi are with Scuola Superiore Sant'Anna, Pisa, Italy.

http://dx.doi.org/10.1364/JOCN.7.00A268 database (TED) that includes information on resource utilization only available in terms of total reserved capacity. Thus, the stateless PCE does not maintain individual label switched path (LSP) state information, which is available only at network nodes. However, this lack of global visibility may impact on the overall efficiency and supported PCE functionalities.

To address this issue, relevant work has been recently provided to define the stateful PCE architecture and its most suitable application scenarios [16,17]. Different with respect to a stateless $\mathrm{PCE}$, a stateful $\overline{\mathrm{PCE}}$ also maintains an LSP state database (LSP-DB), where the PCE stores the attributes of the established LSPs, such as their route through the network, bandwidth/resource usage, switching types, and LSP constraints.

Thus, with respect to the traditional stateless PCE architecture, the stateful functionality enables the PCE to perform, in a more efficient way, a number of use cases requiring fast and effective traffic engineering solutions.

For example, consider the use case of shared path protection (SPP). A stateless PCE could perform SPP by imposing the path computation client (PCC) to provide LSP state information as constraints to the path computation request. However, this may require a huge and timeconsuming exchange of LSP state information through PCE protocol (PCEP), which makes rather awkward such operation. On the other hand, a stateful PCE strongly simplifies SPP by avoiding this exchange of LSP state information every time and directly relying on the information already stored in its LSP-DB.

To further improve PCE functionalities, the stateful PCE has been recently enhanced to also include active functionality. Active functionality enables the PCE to issue recommendations to the network, e.g., to dynamically update LSP parameters.

In this study, the global visibility provided by the stateful functionality together with the active capability of triggering network changes is discussed in the context of several use cases, including impairment-aware path computations in multirate optical networks, recovery solutions, global defragmentation, and dynamic LSP adaptations.

The dynamic LSP adaptation use case is then considered for elastic flexigrid optical networks. Three specific contributions are then provided in this context. 
First, a novel advanced digital signal processing (DSP) monitoring functionality is introduced and experimentally demonstrated. The monitoring functionality enables the coherent receiver to detect minor impairment degradations before experiencing a post-forward-error-correction (FEC) bit error rate (BER). The functionality is implemented in network elements, and an associated messaging capability is designed to send data to the PCE.

Second, a hitless dynamic adaptation technique is proposed and successfully demonstrated. The technique enables a dynamic and hitless adaptation of the applied transmitted low-density parity check (LDPC) code [18, $\underline{19]}$. This way, transmission robustness can be improved upon minor impairment degradations such that no traffic disruption is experienced.

Third, in the case that different spectrum resources need to be reserved to accommodate the LSP adaptations, network reconfiguration is performed, successfully driven by the PCE, augmented with the active functionality.

The three contributions are successfully validated through extensive experimental demonstrations.

\section{Architecture And Protocol Extensions}

To support the active stateful functionality, two main new messages have been introduced [16]:

- Path computation state report (PCRpt). PCRpt is a PCEP message sent by a PCC to a PCE to report the current state and attributes of an LSP, including route, bandwidth, and operational and administrative status. PCRpt is generated, also asynchronously, whenever the LSP state changes.

- Path computation update request (PCUpd). PCUpd is a PCEP message sent by a PCE to a PCC to update LSP parameters, including route, bandwidth, and operational and administrative status.

In addition, to fully support the stateful architecture, several mechanisms have been specifically designed and standardized. The most relevant ones are hereafter reported:

- Advertisement of stateful capability by PCEP speakers, for example, during the initialization phase.

- Maintainance of the LSP state synchronization between PCCs and PCEs. State synchronization is typically performed through PCEP state report messages. However, other means are also enabled. For example, upon restart, a PCE could reacquire the LSP database from a backup copy stored locally.

- Delegation of LSP control from a PCC, as owner of the LSP, to the PCE. The mechanism also defines the revocation on a previously delegated LSP.

The introduction of the stateful functionality, including the global visibility on LSP resources, may introduce scalability issues. However, as reported in $[2,16]$, PCE is not considered to be a solution that is applicable to the entire Internet, and the scale of the domain of visibility has to be adequately dimensioned (as performed for routing areas within service provider networks).

Several effective mechanisms have been introduced to enable a network to be controlled by multiple PCEs (e.g., hierarchical PCE [12]). However, the evolution of the PCE architecture to adopt stateful functionality in the context of multidomain networks is not straightforward (e.g., synchronization issues and visibility of resources at the parent PCE) and it is left for further studies.

\section{Benefits of Active Stateful Architecture}

In addition to the aforementioned shared path protection use case, where the stateful architecture significantly simplifies and speeds up the overall path computation procedure, other use cases can be considered to assess the benefits of this technology. Three relevant examples are described in this section.

\section{A. Impairment-Aware Routing and Spectrum Assignment}

A relevant use case where the stateful architecture can provide significant benefits is impairment-aware routing and spectrum assignment (IA-RSA) path computation, particularly in the context of multirate optical networks.

In multirate networks, optical signals are operated at different bit rates and modulation formats. A typical example refers to $10 \mathrm{~Gb} / \mathrm{s}$ on-off-keying (OOK) that coexists on the same optical infrastructure together with $100 \mathrm{~Gb} / \mathrm{s}$ polarization division multiplexing quadrature phase-shift keying (PM-QPSK). In this scenario, cross-phase modulation (XPM) may induce severe interchannel impairments $[20,21]$. In particular, an OOK $10 \mathrm{~Gb} / \mathrm{s}$ lightpath may induce a very detrimental XPM on the $100 \mathrm{~Gb}$ phase-modulated lightpath. In contrast, the effects of phase-modulated signals on a $10 \mathrm{~Gb} / \mathrm{s}$ OOK lightpath and between lightpaths at the same bit rate or modulation format are typically not so detrimental. That is, a guard band is needed when $10 \mathrm{~Gb} /$ s OOK lightpaths are routed, contiguously in frequency, with $100 \mathrm{~Gb} / \mathrm{s}$ PM-QPSK signals (note that even $300 \mathrm{GHz}$ of unused spectrum may be required as a guard band [17]). On the other hand, no guard band is needed between contiguous $10 \mathrm{~Gb} / \mathrm{s}$ OOK lightpaths or between contiguous $100 \mathrm{~Gb} / \mathrm{s}$ PM-QPSK signals, as shown in Fig. 1(a).

In the case of stateless PCE, as sketched in Fig. 1(b), path computation is not aware of the bit rate and modulation format of existing lightpaths. Thus, the stateless PCE has to consider the worst case scenario and to always include guard bands (e.g., implicitly considered within the frequency slot of each computed lightpath), even if not necessary (e.g., when adjacent lightpaths have the same bit rate and format). This wastes spectrum resources.

In the case of stateful PCE, the LSP-DB can store and maintain the state of the transmission parameters of the existing lightpaths, including the modulation format. This enables the PCE to implement specifically designed IARSA [21,22], which can avoid worst case implementation 
(a)

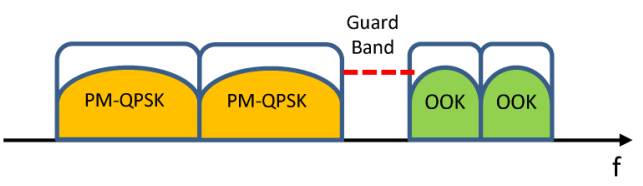

(b)

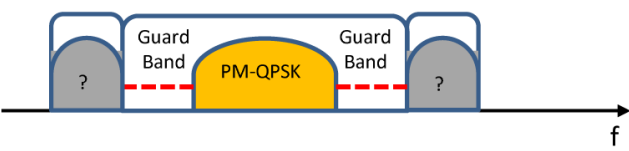

(c)

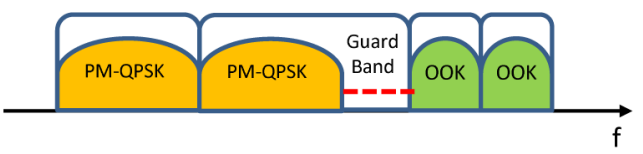

Fig. 1. Example of IA-RSA strategies: (a) ideal RSA; (b) worst case RSA, computed by a stateless PCE; and (c) RSA applying guard bands only if needed, computed by a stateful PCE.

with guard bands always included [as in Fig. 1(b)] and place guard bands only if strictly necessary [as in Fig. 1(c)].

This way, a stateful PCE can provide effective spectrum utilization in the case of lightpath provisioning.

Moreover, thanks to the active functionality, the PCE can perform network reoptimization on reserved spectrum resources by operating on existing lightpaths, in such a way that the wasting of spectrum resources dedicated to guard bands is minimized (e.g., by creating pools of contiguous lightpaths of the same type).

\section{B. Recovery}

A further use case where the active stateful architecture can provide some benefits is during the recovery process, in particular when the PCE is requested to provide the alternative route to the ingress nodes. Figure 2 summarizes the procedure, from the PCE perspective, upon failure occurrence. Three different ingress nodes, operating as PCCs, are assumed to control failed LSPs.

In the first case, shown in Fig. 2(a), a stateless PCE is considered. Upon failure occurrence, the nodes detecting the failure generate routing protocol updates to advertise all network nodes about the failed resources (e.g., a link). The PCE, by listening to routing protocol advertisements, can become aware of the failed resources. Moreover, each upstream node detecting the failure sends a generalized multiprotocol label switching (GMPLS) error message to notify the ingress node about the LSP failure. Each ingress node, operating as a PCC, immediately triggers a path computation request (PCReq) to the PCE. Thus, the stateless PCE receives multiple independent requests for path computations. Upon performance of each path computation, the PCE sends path computation reply (PCRep) messages to every PCC. This concludes the recovery process from the perspective of the stateless PCE architecture (then ingress nodes will perform the signaling over the newly computed path, if successfully identified).

In the second case, shown in Fig. 2(b), a stateful PCE is considered. Different with respect to the previous case of stateless architecture, once the PCE becomes aware of the failed resources, it is immediately able to identify the failed LSPs. Indeed, the route of all established LSPs is stored within the LSP-DB available at the stateful PCE. Thus, the stateful PCE can immediately perform all needed path computations, without waiting for all PCReq messages. Once the PCReq messages are actually received from the involved ingress nodes, the stateful PCE can be ready to reply with PCRep messages. This way, the overall recovery time can be successfully reduced. Moreover, in this case, the path computation can also be performed in a joint (bulk) way, with relevant benefits in terms of path computation efficiency and probability to successfully identify alternative routes to failed LSPs.

In the third case, shown in Fig. 2(c), an active stateful PCE is considered. As in the previous case, once the PCE becomes aware of the failed resources, it is immediately able to identify the failed LSPs and perform bulk path computation. However, different with respect to the previous case of stateful architecture, in the case of active stateful, the PCE is also subsequently able to provide the ingress nodes with alternative routes, without waiting for PCReq messages to be received. Thus, as soon as the bulk path computation is completed, PCUpd messages are immediately sent to the PCCs. This way, the overall recovery time can be further reduced.

\section{Global Defragmentation}

A significant use case particularly suitable for the active stateful architecture consists of network defragmentation.

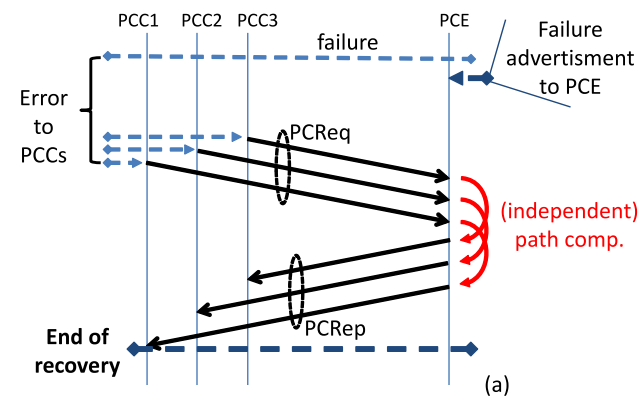

(a)

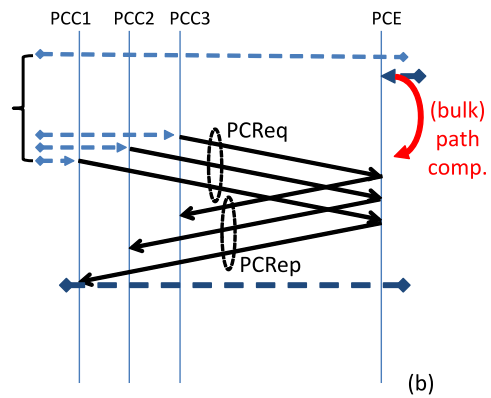

(b)

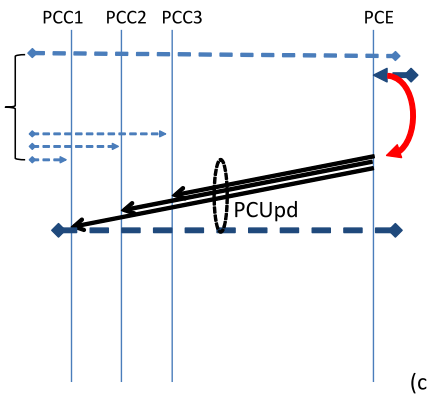

(c)

Fig. 2. Recovery procedure from the PCE perspective in the case of (a) stateless PCE, (b) stateful PCE, and (c) active stateful PCE. 
In the case of a stateless $\mathrm{PCE}$, the reoptimization of network resource usage can be performed only upon request from a PCC. In particular, a PCC has to send a request to the PCE together with detailed route and bandwidth information of the LSPs that are requested to be concurrently optimized. This implies the use of the inefficient and timeconsuming aforementioned PCEP procedure. Moreover, and most important, the PCC has to be able to determine when and which LSPs should be optimized. However, this capability is typically not available at PCCs, making this procedure extremely inefficient.

On the other hand, the capability provided by the stateful architecture to exploit both unreserved traffic engineering resources (maintained at the PCE TED) and LSP routes (maintained at the PCE LSP-DB) enables the PCE to evaluate the fragmentation level of the controlled optical infrastructure. Specifically designed defragmentation algorithms (e.g., [23-25]) can be applied to assess potential benefits provided by network reoptimization procedures. These evaluations can be performed upon network events that potentially induce network defragmentation, such as tear-down of one/many LSPs, LSPs rerouting upon failure occurrence, or after failure repair operation [26]. In addition, the potential benefits of network reoptimization could be evaluated also asynchronously, i.e., periodically or during off-peak traffic conditions.

Then, when the active functionality is introduced, the PCE is also able to directly trigger network optimization. Indeed, if the potential benefits highlighted by the evaluation phase are considered adequate, the active PCE can operate on selected/all LSPs, by providing through PCEP PCUpd messages the new LSP attributes. For example, the PCE can schedule and directly apply specific sequences of operations, aiming at limiting or avoiding dead-lock conditions and traffic disruption.

\section{Dynamic LSP Adaptation}

A special case of the network reconfiguration described in the previous subsection is the adaptation due to dynamic requests on specific LSPs. In the case that the request of a new LSP is sent to the PCE and no network resources are available to satisfy it, a stateless PCE would simply reject the provisioning request. Similarly, the case of dynamic adaptation of some attributes of an existing LSP may require the intervention of the PCE. For example, upon degradation of transmission performance, an LSP could rely on different options to improve transmission robustness, including modulation format adaptation, LDPC code adaptation, or even rerouting. However, the choice among these options may be complex and related to the need and availability of additional spectrum resources (e.g., to preserve the committed information rate).

However, the PCC or the stateless PCE, due to the lack of global visibility on the utilized LSP resources, is not able to perform all possible adaptation operations. For example, if contiguous spectrum resources are not available, a stateless PCE would simply reject an adaptation request.
On the other hand, when the active stateful PCE architecture is considered, a reconfiguration limited to a specific LSP or to a portion of the network (e.g., involving just a few adjacent established LSPs) may be considered to subsequently satisfy the specific provisioning or adaptation request. Different with respect to global optimizations, this dynamic reconfiguration does not require complex and time-consuming algorithms considering the whole network optimization. Indeed, fast reconfiguration techniques could be adopted to address just the considered request.

This specific use case is experimentally demonstrated in this paper.

\section{Active Stateful PCE Implementation}

The architecture of the implemented active stateful PCE is shown in Fig. 3.

The PCE architecture relies on the TED and, as introduced by the stateful technology, also the LSP-DB storing the state of all established LSPs. Moreover, the PCE includes the actual path computation engine, here called Active Solver. The Active Solver runs the path computation algorithms, also relying on transmission models that account for expected and actual (i.e., collected through monitoring) physical impairments. Then, path computation includes the route (i.e., the sequence of node and links), the suggested spectrum (i.e., the central frequency $n$ and the number of frequency slices $m$ of the ITU-T flexible grid based on $12.5 \mathrm{GHz}$ slice granularity [27]), and the suggested physical parameters of the source and destination transponders that properly achieve the optical reach of the selected path. Path computation can also be performed by elaborating a scheduling strategy where a combination of rerouting operations of existing paths is considered [15]. To this extent, the PCE possibly considers nondisruptive defragmentation operations, including the push-pull technique that implements the hitless shift of lightpaths [28]. The computed strategy can be applied thanks to the active functionality. The Action Solver then interacts with the Action Handler, an internal module specifically introduced to address bundle and synchronization aspects within a sequence of operations. The Action Handler module is

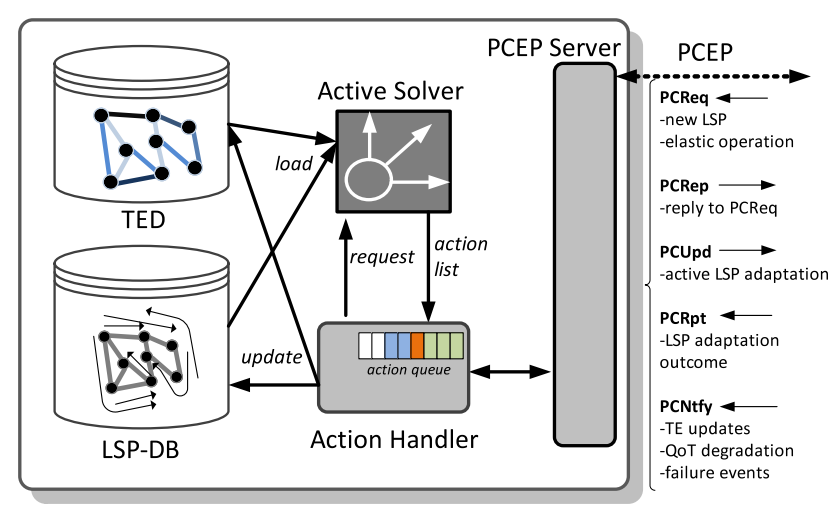

Fig. 3. Active stateful PCE architecture. 
utilized to synchronize reoptimization operations, thus handling deadlock conditions and enabling the defragmentation of the network resources contiguous to the considered LSP. The Action Handler receives the following PCEP messages from the PCEP server:

- PCReq for new LSPs;

- PCReq updates for existing LSPs (bit rate modification);

- PCRpt about configuration outcome over delegated LSPs;

- PCNtf about TE updates; and

- PCNtf about quality of transmission (QoT) degradations or network events.

In particular, PCReq messages are forwarded to the Action Solver for path computation/update. PCRpt and TE update PCNtf are used to update the LSP-DB and the TED. PCNtfs are used to handle TE updates and, as considered in this study, also specific QoT variations. PCNtfs are then used to drive LSP adaptation/rerouting. The latter case triggers update submission to the Active Solver.

The Active Solver enables multi-action path computation. The considered output actions of the Action Solver, besides the NO-PATH option, are the following:

- new LSP setup;

- adapt existing LSP;

- shift existing LSP; and

- reroute existing LSP.
The Adapt action is referred either to elastic operations (i.e., subcarrier switch on/off, baud rate variation [15]) or transmission parameter variation (e.g., modulation format $[4,29]$, code [1]) to be enforced on the existing LSP without rerouting actions. The Shift action is referred to the defragmentation operation and considers the push-pull technique [28], enabling the hitless frequency shift of the LSP.

For each single path computation request/update, the Active Solver returns a list of actions. Such actions may need to wait until some previous ones are actually configured on the data plane. For example, in order to make space for a new LSP setup, a PCReq for a new LSP submitted to the Active Solver could generate two Shift actions followed by a New LSP action. In this case, the shift of the two adjacent channels has to be completed before the setup of the new LSP. To this purpose, each action is ordered and includes the list of possible dependent actions that need to be terminated before its execution. The output actions are inserted in the Action Queue and processed by the Action Handler. Each action is removed from the queue only once it has been successfully established in the network and its outcome has been received by the PCE (e.g., a PCRpt enclosing the same LSP object).

\section{Demonstration of Elastic Operation for Code ADAPTATION}

The active stateful PCE architecture described in Section IV has been applied to a flexigrid elastic optical network testbed shown in Fig. 4. The testbed includes bandwidth-variable optical cross connects (BV-OXCs, also

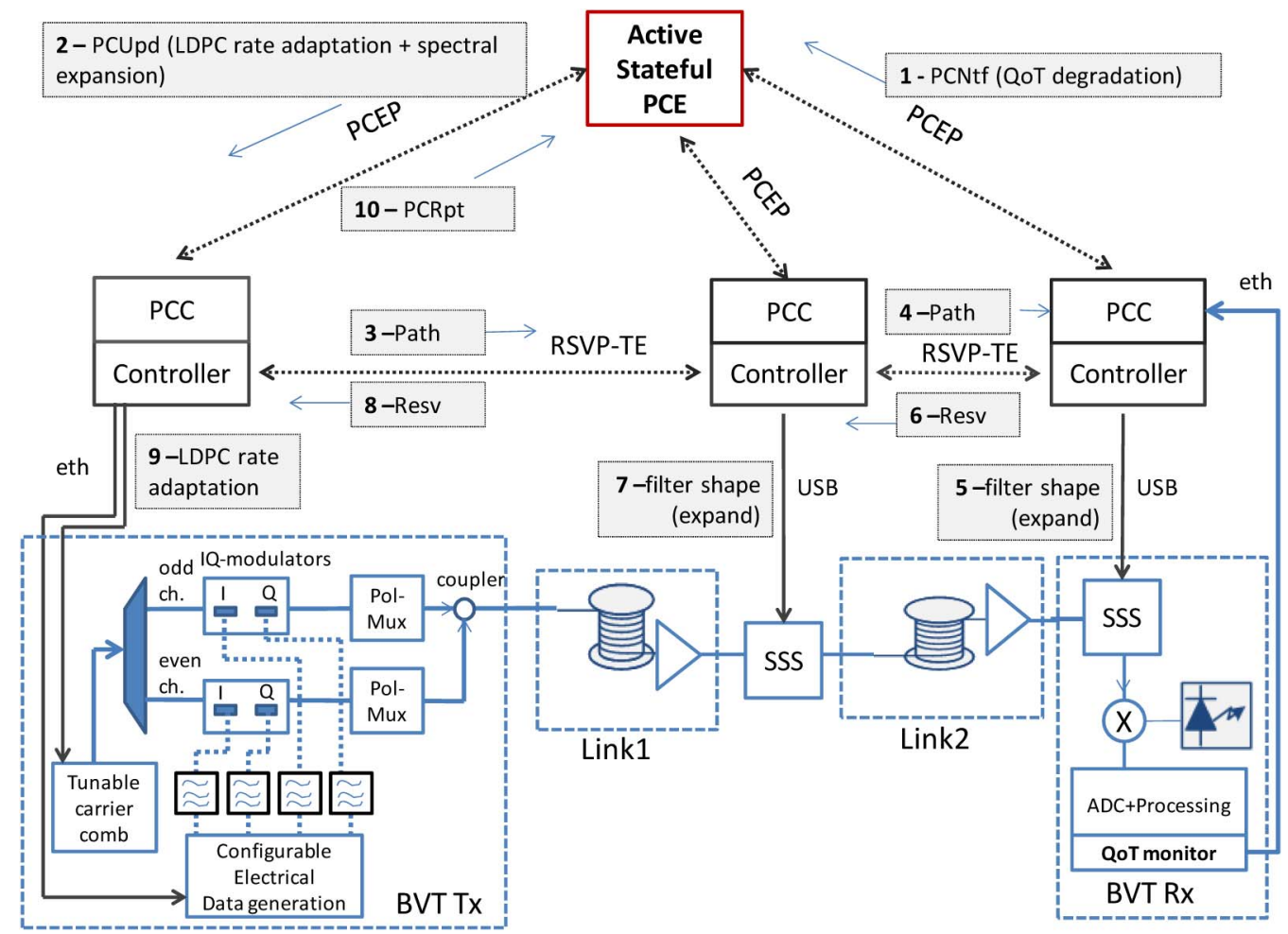

Fig. 4. Experimental flexigrid optical network testbed. 
called spectrum selective switches-SSSes) and bandwidth variable transponders (BVTs) implementing up to $1 \mathrm{~Tb} / \mathrm{s}$ transmission over $200 \mathrm{GHz}$ through seven carriers at up to $160 \mathrm{~Gb} / \mathrm{s}$ PM-QPSK (40 Gbaud). The transmission relies on a time-frequency-packing (TFP) technique through configurable low-density parity-check (LDPC) coding with coherent detection [30]. Control plane includes GMPLS controllers running $\overline{R S V P-T E}$ for resource reservation and PCEP for path computation and notification toward the central active stateful PCE. The GMPLS controllers located at the source/destination transponders are able to dynamically configure the number of generated subcarriers, the tunable lasers, the modulation format, and the LDPC code through dedicated Ethernet, universal serial bus (USB), and serial interfaces. Moreover, at the destination node, QoT optical parameters computed by the DSP at the receiver are collected each $15 \mathrm{~s}$ for monitoring purposes. The node controller locally compares the QoT parameter values to predefined threshold values, triggering alarms to the PCE when thresholds are exceeded. The node controllers located at intermediate nodes implement GMPLS-driven BV-OXC cross connections and a filter shaping configuration. Node controllers and the PCE are implemented in $\mathrm{C}++$ on Linux boxes and connected by means of gigabit Ethernet interfaces.

\section{A. LDPC Code Performance and Optical Monitoring}

Adjustments to the reserved spectrum resources of existing lightpaths can be operated either to cope with requests for additional bit rate or to deal with the degradation of transmission performance. The implemented active stateful PCE has been here applied to address the latter case. A set of multiple LDPC codes with different overhead are available, e.g., of type 8/9 (8 information bits out of 9 transmitted).

The optimum LDPC code is computed by the PCE and set according to link condition and the desired reach. As distance increases, impairments due to optical signal-tonoise ratio (OSNR) degradation and nonlinear effects arise, limiting the maximum achievable information rate. Figure 5 shows the net information rate as a function of the desired reach for the considered superchannel. For a distance such as $3000 \mathrm{~km}$, low overhead is required (i.e., low redundancy LDPC code such as 9/10 or 8/9 can be used), guaranteeing a $1 \mathrm{~Tb} / \mathrm{s}$ net information rate. As distance increases, OSNR degradation and the impact of nonlinear effects require more robust coding (i.e., 5/6 or $3 / 4)$, thus reducing the net information rate.

Figure 6 shows an example of typical OSNR evolution as propagation distance increases and multiple erbium-doped fiber amplifiers (EDFAs) are traversed. TFP employs DSP algorithms to retrieve the transmitted data. Advanced monitoring functionalities have been implemented to provide useful information about the quality of the received signal, based on observation of uncorrected BER (preFEC) and the variance of the acquired samples. In particular, sample variance is deeply related to OSNR and can be

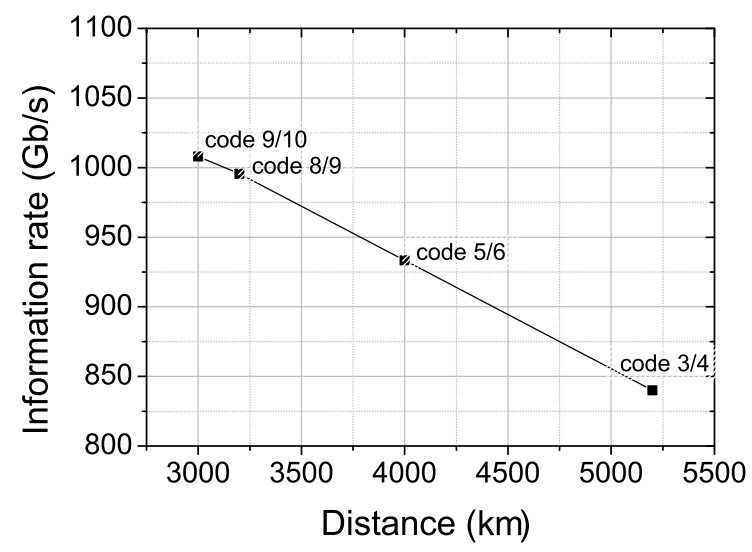

Fig. 5. Net information rate for the seven-carrier superchannel as a function of the optical reach. As the signalling rate is maintained at $40 \mathrm{Gbaud}$, code redundancy has to be increased with distance to cope with transmission impairments, thus reducing the net information rate.

useful for monitoring OSNR variations. Therefore, dynamic LDPC code adaptation can be performed in the case of OSNR degradation.

Figures 7(a)-7(c) show the measured variance of the acquired samples as a function of OSNR. Moreover the figures show, for different codes, the OSNR range of use where the applied code can successfully operate (solid line). Note that the variance does not depend on the applied LDPC code. For each code, an OSNR threshold is identified at a certain margin (i.e., $1 \mathrm{~dB}$ ) from the minimum OSNR required for error-free operation. Monitoring of variances then reveals any OSNR degradation and indicates whether the working-limit condition is approaching for the code in use. Therefore, such a monitoring system can predict OSNR degradation in advance, before reaching the working-limit condition. If the OSNR degradation exceeds the threshold and remains within the margin (e.g., 0.07 for 8/9 coding), no post-FEC BER degradation is experienced, but a warning alarm is locally triggered to switch to a more robust code before post-FEC errors occur.

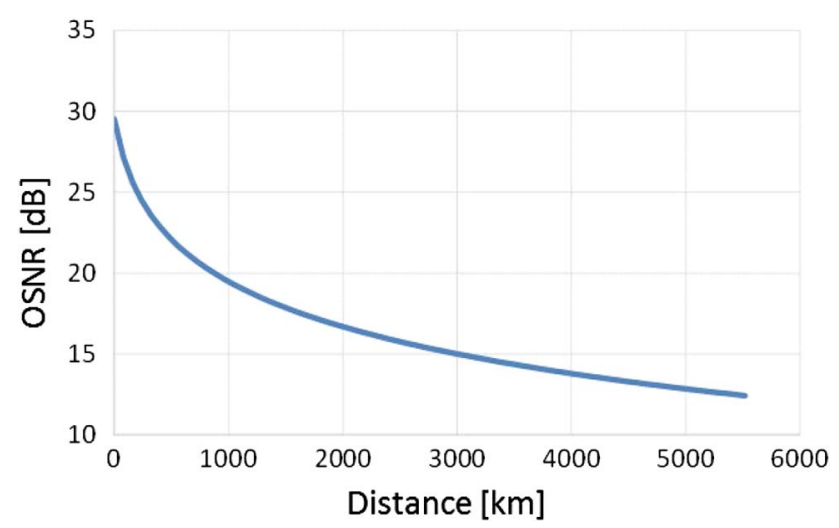

Fig. 6. Example of OSNR evolution along a transmission path, as a consequence of cumulated amplified spontaneous emission due to the traversed EDFAs. 

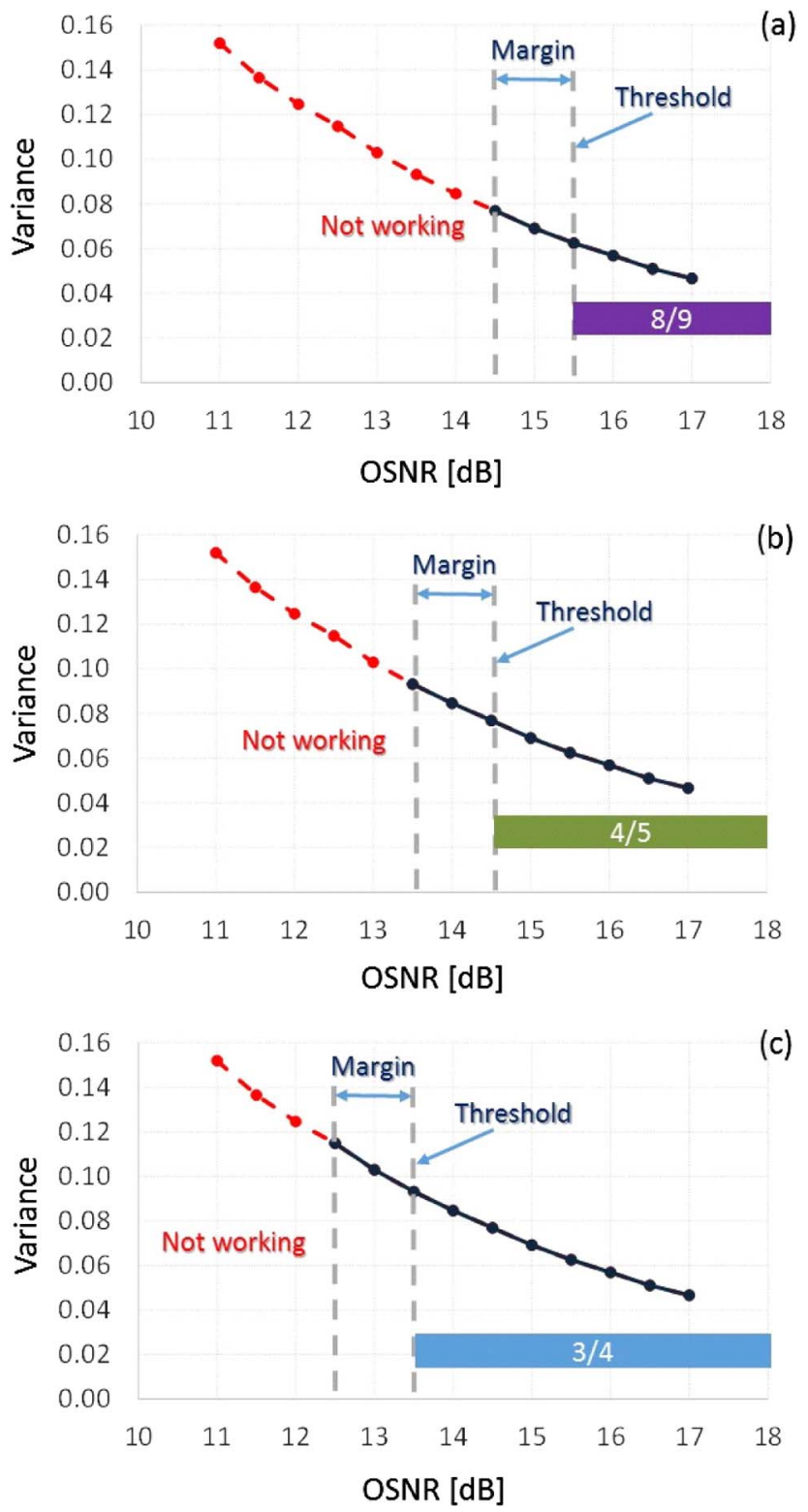

Fig. 7. Variance of the acquired samples as a function of the OSNR. For different codes [(a) 8/9, (b) 4/5, and (c) 3/4] the OSNR range of use where the applied code can successfully operate is reported (solid line). The OSNR warning threshold is set opportunely at a certain margin from the working-limit condition. Then, the corresponding value of variance is established as the threshold for monitoring operation.

\section{B. PCE-Driven Hitless Adaptation}

Figure 8 reports the Wireshark capture of the control plane packets collected at the active stateful PCE (IP address 10.0.0.49) exchanged with source (IP 10.0.0.1) and destination (IP 10.0.0.2) nodes. In addition to handshake, a $1 \mathrm{~Tb} / \mathrm{s}$ LSP is computed by the PCE having $8 / 9$ coding and established in the testbed (see PCReq, PCRep, and TE Ntf messages, packets 31,33 , and 35 , respectively).

After the LSP establishment, OSNR degradation is induced in the path by increasing the attenuation of a

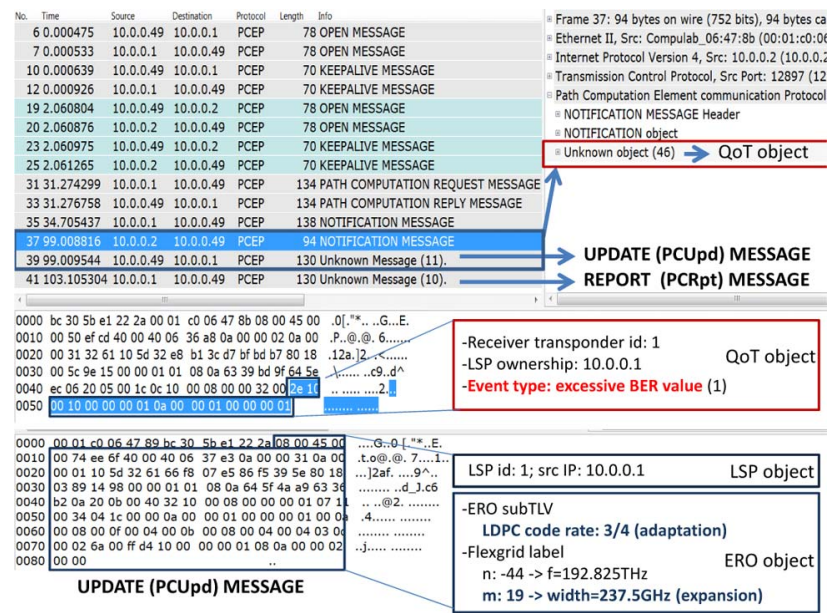

Fig. 8. Capture of PCEP messages enabling dynamic code adaptation.

variable optical attenuator (VOA) at the input port of the EDFA placed on Link2 of Fig. 4. According to the aforementioned predefined policies, a notification message (i.e., PCEP Ntf extended with a novel QoT object; see Fig. 8, packet 37 ) is sent to the PCE to notify of the LSP degradation and trigger the improvement of transmission robustness. The QoT object identifies the affected LSP and the alarm event. In particular, the QoT alarm indicates an excessive pre-FEC BER value. The PCE, based on information retrieved from the LSP-DB, elaborates the parameters to adapt the LSP, considering network resources, modulation format, coding, or a combination of the above. In this experiment, the PCE computes LDPC coding adaptation from $8 / 9$ to $4 / 5$. To preserve the information rate, an additional carrier is also activated. This imposes an increase of the occupied spectrum resources, from 200 to $237.5 \mathrm{GHz}$. To make room for such an increase, the PCE has the capability to first trigger network de-fragmentation, as we showed in [15]. To improve transmission robustness, the PCE sends a PCUpd message to increase the reserved spectrum resources of the degraded LSP and apply the code adaptation (see Fig. 8, packet 39). A novel explicit routing object (ERO) subtype-length-value (SubTLV) is enclosed to specify the updated LDPC code at the source node transmitter. The source node runs the RSVP-TE signaling (using a make-beforebreak mechanism) to adapt the BV-OXC filter configurations along the path.

Then, the new coding is activated in the transmitted data via software configuration of the configurable electrical data generation block of the transmitter. Within the preamble of each data block, a specifically introduced 3 bit field is configured as part of the overhead to communicate to the receiver the applied $4 / 5$ coding. This way, the receiver becomes aware of the code to apply. It processes incoming data through the new coding, successfully supporting the more robust transmission, which aims at preventing post-FEC BER degradation. Code adaptation is performed with no traffic disruption.

At the end of the procedure, a PCRpt message is sent to the active PCE to inform about its outcome (packet 41). 


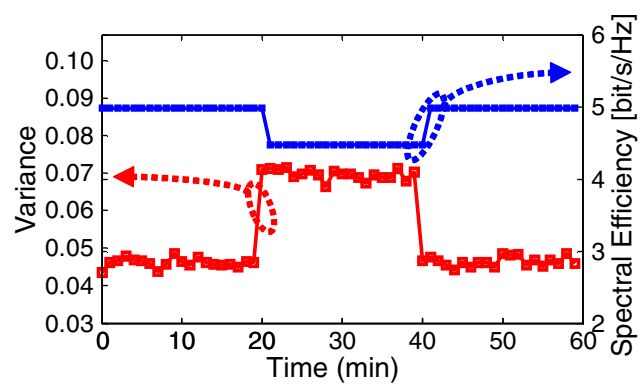

Fig. 9. Monitoring of sample variance versus time and spectral efficiency. OSNR degradation is intentionally introduced (minute $20)$ to demonstrate dynamic LDPC code adaptation. As the measured variance exceeds the predefined threshold for code 8/9 (0.06), a warning is triggered and more robust code $4 / 5$ is immediately adopted. Consequently, spectral efficiency is reduced due to larger overhead. After OSNR degradation is removed (minute 40), the initial condition is dynamically restored.

The time needed to complete the whole adaptation is around $4 \mathrm{~s}$. This value is mainly due to the time needed to configure the BV-OXC filters by means of the BV-OXC proprietary software tool.

In the case that spectrum reservation is not modified, and only the hitless code adaptation procedure is performed, only a few milliseconds are required.

Figure 9 reports the collected variance statistics before and after degradation is induced. The figure also shows the related spectral efficiency of the transmitted data. As degradation occurs, the variance increases above the predefined threshold for code 8/9 (0.06) and spectral efficiency is reduced due to the more robust applied code. Moreover, after a certain time, the source of degradation is removed (i.e., the VOA gain is reset to the initial value) and the link condition returns to the initial state. Consequently, the observed variance returns below the predefined threshold for code $8 / 9$. PCE can then restore the initial 8/9 code, as the higher redundancy of $4 / 5$ is no longer required, thus increasing spectral efficiency back to the initial value.

\section{CONCLUSIONS}

In this study, the active stateful PCE architecture has been described and discussed considering several different use cases.

In multirate optical networks, the active stateful PCE enables effective spectrum utilization by minimizing guard bands during provisioning and reoptimization. In PCEbased recovery, the active functionality can speed up the overall PCEP procedure while guaranteeing effective bulk path computations. In global defragmentation, the active PCE has the capability to evaluate and subsequently apply effective defragmentation strategies. In elastic optical networks, the active PCE can provide dynamic adaptations to transmission parameters and attributes, possibly operating on other adjacent LSPs.

This last use case was then experimentally demonstrated.
A new advanced monitoring functionality exploiting DSP parameters was first introduced and experimentally demonstrated in a flexigrid optical network testbed including a multicarrier terabit LSP. The DSP parameters were used to detect impairment degradations before the BER was affected.

A new dynamic LDPC code adaptation technique was then introduced and validated to dynamically adapt the transmission robustness. Hitless adaptation of the applied LDPC coding was achieved in only a few milliseconds with no traffic disruption.

Moreover, the technique has been applied within a more complex procedure encompassing BV-OXC reconfigurations, successfully triggered by the active functionality of the implemented PCE.

\section{ACKNOWLEDGMENTS}

This paper is an extended version of the work presented in [1]. This work was partially supported by the EU-FP7 IDEALIST project under grant agreement number 317999.

\section{REFERENCES}

[1] F. Cugini, F. Paolucci, F. Fresi, G. Meloni, G. Berrettini, N. Sambo, A. Giorgetti, T. Foggi, L. Poti, and P. Castoldi, "Benefits of active stateful PCE for flexgrid networks," in Optical Fiber Communication Conf., 2014, paper Th3I.1.

[2] A. Farrel, J. P. Vasseur, and J. Ash, "A Path Computation Element (PCE)-based architecture," IETF RFC 4655, Aug. 2006.

[3] J. Vasseur and J. Le Roux, "Path Computation Element (PCE) Communication Protocol (PCEP)," IETF RFC 5440, Mar. 2009.

[4] F. Cugini, G. Meloni, F. Paolucci, N. Sambo, M. Secondini, L. Gerardi, L. Poti, and P. Castoldi, "Demonstration of flexible optical network based on path computation element," $J$. Lightwave Technol., vol. 30, no. 5, pp. 727-733, Mar. 2012.

[5] R. Munoz, R. Casellas, R. Martinez, and R. Vilalta, "PCE: What is it, how does it work and what are its limitations?" J. Lightwave Technol., vol. 32, no. 4, pp. 528-543, Feb. 2014.

[6] O. de Dios, V. Lopez, M. Cuaresma, F. Munoz, M. Chamania, and A. Jukan, "Coordinated computation and setup of multilayer paths via inter-layer PCE communication: Standards, interoperability and deployment," IEEE Commun. Mag., vol. 51, no. 12, pp. 144-154, Dec. 2013.

[7] M. Chamania, M. Drogon, and A. Jukan, "An open-source path computation element (PCE) emulator: Design, implementation, and performance," J. Lightwave Technol., vol. 30, no. 4, pp. 414-426, Feb. 2012.

[8] Y. Zhao, J. Zhang, Y. Ji, and W. Gu, "Routing and wavelength assignment problem in PCE-based wavelength-switched optical networks," J. Opt. Commun. Netw., vol. 2, no. 4, pp. 196-205, Apr. 2010.

[9] J. Ahmed, C. Cavdar, P. Monti, and L. Wosinska, "A dynamic bulk provisioning framework for concurrent optimization in PCE-based WDM networks," J. Lightwave Technol., vol. 30, no. 14, pp. 2229-2239, July 2012.

[10] Q. Zhang, M. Hasan, X. Wang, P. Palacharla, and M. Sekiya, "Survivable path computation in PCE-based multi-domain 
networks," J. Opt. Commun. Netw., vol. 4, no. 6, pp. 457-467, June 2012.

[11] F. Paolucci, N. Sambo, F. Cugini, A. Giorgetti, and P. Castoldi, "Experimental demonstration of impairment-aware PCE for multi-bit-rate WSONs,” J. Opt. Commun. Netw., vol. 3, no. 8, pp. 610-619, Aug. 2011.

[12] F. Paolucci, F. Cugini, A. Giorgetti, N. Sambo, and P. Castoldi, "A survey on the path computation element (PCE) architecture," IEEE Commun. Surv. Tutorials, vol. 15, no. 4, pp. 1819-1841, 2013.

[13] A. Castro, R. Martinez, R. Casellas, L. Velasco, R. Munoz, R. Vilalta, and J. Comellas, "Experimental assessment of bulk path restoration in multi-layer networks using PCE-based global concurrent optimization," J. Lightwave Technol., vol. 32 , no. 1, pp. 81-90, Jan. 2014.

[14] R. Munoz, R. Casellas, R. Vilalta, and R. Martinez, "Dynamic and adaptive control plane solutions for flexi-grid optical networks based on stateful PCE," J. Lightwave Technol., vol. 32, no. 16, pp. 2703-2715, 2014.

[15] A. Castro, F. Paolucci, F. Fresi, M. Imran, B. Bhowmik, G. Berrettini, G. Meloni, A. Giorgetti, F. Cugini, L. Velasco, L. Poti, and P. Castoldi, "Experimental demonstration of an active stateful PCE performing elastic operations and hitless defragmentation," in 39th European Conf. and Exhibition on Optical Communication (ECOC), Sept. 2013.

[16] E. Crabbe, J. Medved, I. Minei, and R. Varga, "PCEP extensions for stateful PCE," IETF draft, 2014 [Online]. Available: https://tools.ietf.org/html/draft-ietf-pce-stateful-pce-10.

[17] X. Zhang and I. Minei, "Applicability of stateful path computation element (PCE)," IETF draft, 2014 [Online]. Available: https://tools.ietf.org/html/draft-ietf-pce-stateful-pce-app-03.

[18] I. Djordjevic, M. Cvijetic, and C. Lin, "Multidimensional signaling and coding enabling multi-Tb/s optical transport and networking: Multidimensional aspects of coded modulation," IEEE Signal Process. Mag., vol. 31, no. 2, pp. 104-117, Mar. 2014.

[19] L. Schmalen and A. Leven, "Status and recent advances on forward error correction technologies for lightwave systems," in 39th European Conf. and Exhibition on Optical Communication (ECOC), Sept. 2013.

[20] A. Bononi, M. Bertolini, P. Serena, and G. Bellotti, "Crossphase modulation induced by OOK channels on higher-rate DQPSK and coherent QPSK channels," J. Lightwave Technol., vol. 27, no. 18, pp. 3974-3983, Sept. 2009.
[21] N. Sambo, M. Secondini, F. Cugini, G. Bottari, P. Iovanna, F. Cavaliere, and P. Castoldi, "Modeling and distributed provisioning in 10-40-100 Gb/s multirate wavelength switched optical networks," J. Lightwave Technol., vol. 29, no. 9, pp. 1248-1257, May 2011.

[22] H. Beyranvand and J. Salehi, "A quality-of-transmission aware dynamic routing and spectrum assignment scheme for future elastic optical networks," J. Lightwave Technol., vol. 31, no. 18, pp. 3043-3054, Sept. 2013.

[23] D. Geisler, Y. Yin, K. Wen, N. Fontaine, R. Scott, S. Chang, and S. Yoo, "Demonstration of spectral defragmentation in flexible bandwidth optical networking by FWM," IEEE Photon. Technol. Lett., vol. 23, no. 24, pp. 1893-1895, Dec. 2011.

[24] R. Wang and B. Mukherjee, "Provisioning in elastic optical networks with non-disruptive defragmentation," J. Lightwave Technol., vol. 31, no. 15, pp. 2491-2500, Aug. 2013.

[25] H. Choi, T. Tsuritani, and I. Morita, "Feasibility demonstration of spectrum defragmentation in flexigrid optical network employing multi-format and multi-rate transmitter/ receiver," Electron. Lett., vol. 49, no. 5, pp. 361-362, Feb. 2013.

[26] L. Velasco, F. Paolucci, L. G. Renom, A. Aguado, F. Cugini, P. Castoldi, and V. Lopez, "First experimental demonstration of ABNO-driven in-operation flexgrid network re-optimization," in Optical Fiber Communication Conf., 2014, paper Th5A.3.

[27] "Draft revised G.694.1 version 1.3," unpublished ITU-T Study Group 15, Question 6.

[28] F. Cugini, F. Paolucci, G. Meloni, G. Berrettini, M. Secondini, F. Fresi, N. Sambo, L. Poti, and P. Castoldi, "Push-pull defragmentation without traffic disruption in flexible grid optical networks," J. Lightwave Technol., vol. 31, no. 1, pp. 125-133, Jan. 2013.

[29] G. Meloni, F. Paolucci, N. Sambo, F. Cugini, M. Secondini, L. Gerardi, L. Poti, and P. Castoldi, "PCE architecture for flexible WSON enabling dynamic rerouting with modulation format adaptation," in European Conf. on Optical Communication (ECOC), Sept. 2011, paper Tu.5.K.3.

[30] N. Sambo, G. Meloni, F. Paolucci, F. Cugini, M. Secondini, F. Fresi, P. Pot, and L. Castoldi, "Programmable transponder, code and differentiated filter configuration in elastic optical networks," J. Lightwave Technol., vol. 32, no. 11, pp. 20792086, June 2014. 